\title{
Features of surface enhanced Raman scattering in the systems with «hot spots»
}

\author{
E.V. Solovyeva*, D.A. Khazieva, and A.S. Denisova \\ Saint-Petersburg State University, Institute of Chemistry, 199034 Saint-Petersburg, Russia
}

\begin{abstract}
In this work we demonstrate the features of SERS on the substrates with «hot spots» on the example of system «diaminostilbene colloidal silver». We found that «hot spots» forming on aggregated nanoparticles exist on the metal substrates only at low concentration of ligand. This effect caused by the gradual filling of first monolayer by adsorbate molecules. Significantly higher enhancement factor is obtained for substrates with «hot spots», for which the participation of resonance processes in the formation of SERS signal is revealed also.
\end{abstract}

Surface enhanced Raman scattering (SERS) is the rapidly developing technique of vibrational spectroscopy based on the phenomenon of Raman signal amplification for molecules adsorbed on metallic nanostructured surface or nanoparticles. Recently, the special interest was devoted to the SERS investigation on substrates which have "hot spots" - areas where the surface plasmons are localized [1]. One consequence of this effect is more significant amplification of optical signal from the molecules placed in the "hot spots", that is especially valuable for vibrational spectroscopy, in particular, for SERS [2].

The presence of "hot spots" is typical for substrates with closely spaced nanoparticles where the interparticle gap does not exceed a few nanometers. One of the methods for obtaining such substrates is linking of nanoparticles by chemical modifiers. Usually, the modifiers are compounds with two spatially separated functional groups, where each of them is capable to react with metal surface. In this work we have shown the features of SERS on the substrates with "hot spots" obtained by linking silver nanoparticles via 4,4'diaminostilbene (DAS) molecules. DAS has conjugated system and two amino groups located on the opposite ends. Due to this structure DAS is attractive for creation the substrates with "hot spots".

For SERS spectra of DAS it was found that their intensity changes in non-monotonic way with the increasing of ligand concentration. Also, the profile of SERS spectra transforms dramatically at concentration equal to $5 \cdot 10^{-5} \mathrm{M}$ (Fig. 1).

The observed behavior of DAS SERS spectra is caused by two main factors associated with the gradual filling of surface area by adsorbate molecules. The one of them is "the effect of first monolayer" when the greatest enhancement of Raman signal arises for molecules from the first monolayer. The next is the existence of "hot spots" in silver colloid solely at low concentration of DAS. In this case the partial coverage of the surface is

\footnotetext{
* Corresponding author: solovyeva elena v@gmail.com
} 
reached only. The last supposition was confirmed by scanning electron microscopy and UV-VIS spectroscopy.

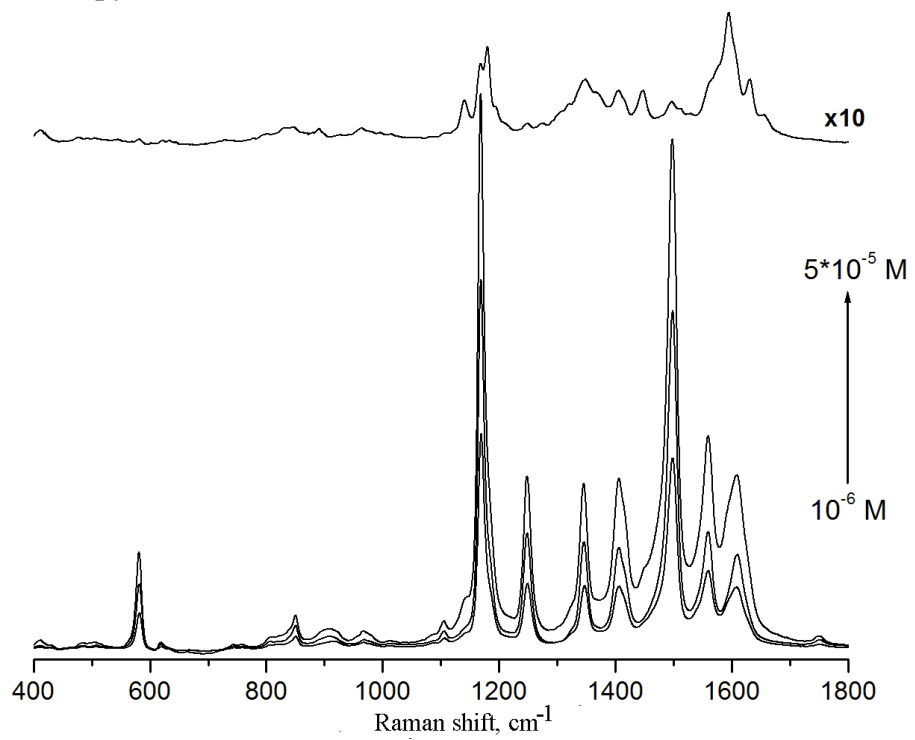

Fig. 1. SERS spectra of DAS adsorbed on silver nanoparticles at various concentrations; the spectrum at $\mathrm{C}_{\mathrm{DAS}}=5 \cdot 10^{-5} \mathrm{M}$ is multiplied by 10 and shifted along axis $\mathrm{y}$.

This work was supported by the research Grant (number 12.38.235.2014) of SaintPetersburg State University. The authors acknowledge the Research Center for X-ray Diffraction Studies and Nanotechnology Interdisciplinary Center of Saint-Petersburg State University.

\section{References}

1. J. Kubackova, I. Izquierdo-Lorenzo, D. Jancura, P. Miskovsky, S. Sachez-Cortes, Phys. Chem. Chem. Phys. 16, 11461 (2014)

2. E.Le Ru, P. Etchegoin, Principles of Surface-Enhanced Raman Spectroscopy and related plasmonic effects (Amsterdam, Elsevier, 2009) 